\title{
Autonomous Fault Tolerant Multi-Robot Coordination for Object Transportation Based on Artificial Immune System
}

\author{
Muhammad Tahir Khan, Clarence W. de Silva, Fellow, IEEE
}

\begin{abstract}
An efficient coordination strategy is required in order to realize an efficient and autonomous multi-robot cooperative system. This paper presents an approach for coordination among robots prior to cooperative object transportation. This paper does not address the coordination that is required during cooperation, which has been researched by others. In the present paper, fault tolerant coordination is achieved using methodology of Artificial Immune System. The approach developed here is based on binding affinity between an antibody and an antigen, and the structure of antibody in a Human Immune System. The developed methodology is verified through physical experiments.
\end{abstract}

Index Terms-multi-robot systems, communication, coordination, artificial immune system

\section{INTRODUCTION}

$\mathrm{W}$ ITH the rapid progress of robotic technologies, the use of multiple robots working together for material transportation, cooperative assembly, and so on is rapidly becoming a reality. There are many advantages of multi-robot cooperative systems, for example: improved system performance, greater efficiency, better fault tolerance, robustness, more reliability, and cost effectiveness. It is generally known that even when the individual robots possess a limited level of intelligence, the overall intelligence can significantly improve in a group of robots when they can interact with each other. A multi-robot system is a set of autonomous robots, working together to perform a desired task. The robots are typically "intelligent" to some extent. Every robot in the group has its own belief, intention and actions. They sense changes in the environment and other robots and relate this information to their actions. The robots can cooperate, communicate and compete with each other to some extent.

Manuscript received November 15, 2008. This work was supported in part by research grants from the National Science and Engineering Research Council (NSERC) of Canada, The Canada Foundation for Innovation (CFI),Canada Research Chair (CRC) program, and Higher Education Commission (HEC) Government of Pakistan

The authors are with the Department of Mechanical Engineering, University of British Columbia, Vancouver, BC, V6T 1Z4, Canada (email: mkhan@mech.ubc.ca; desilva@mech.ubc.ca; phone: +1 604822 4850; Fax $+16048273524$
A useful application of a multi-robot system is object transportation where several robots move an object to a goal location while avoiding collision with obstacles. A system of object transportation has many applications; for example in assembly, construction, material handling, search and rescue, and space exploration.

Previous research has commonly assumed the number of robots that is required to execute a particular transportation task [1-5], which makes the system less autonomous. An autonomous system must by itself determine the number of robots needed to transport a part. This requires coordination among the robots even before the cooperation starts. In the present paper, a biologically inspired solution is presented to achieve this type of autonomous multi-robot coordination.

Biological information systems are autonomous and distributed. They are robust and present a valuable source of ideas for problem solution in object transportation. Inspired by biological systems, researchers have proposed numerous solutions for the tasks under consideration using neural networks, genetic algorithm, human inspired fuzzy logics, insect behavior inspired foraging, and swarm robotics. In the present paper a biologically inspired solution is developed based on human immune system, to realize an autonomous fault-tolerant multi-robot coordination system which can work in unknown and dynamic environments, in the absence of central knowledge.

\section{RELATED WORK}

Some significant work has been done in competitive and cooperative multi-robot systems. There are two subgroups who carry out research in this area, one from the engineering perspective and the other from the biological perspective.

Mataric, et al. [1] studied the cooperative multi-robot object pushing problem. The paper explored the idea of communication in task sharing between two commercially available autonomous six legged robots. The control strategy was a reactive one based on immediate sensory information. Parker $[2,3]$ proposed the software architecture ALLIANCE, which facilitates the fault tolerant cooperative control of a team of mobile robots performing hazardous waste cleanup and box pushing.

Wang and de Silva [4] proposed a new multi-agent architecture to realize effective cooperation between multiple 
autonomous robots, in carrying out a task. In this architecture, reinforcement learning (RL) and genetic algorithms (GA) are combined to learn a cooperative strategy. They used three robots to push a box to a goal location. Liu, et al. [5] investigated the evolution of multi-agent cooperative behavior in an object pushing problem. A genetic algorithm was employed as a machine learning tool. Gao and Wei [6] proposed an immune agent model by combining an artificial immune system with agent technology. Robots in the model perform self-determinated cooperation in a new environment without knowledge of the variety or tasks. Lau, at al. [7] proposed an immunity based control frame work, which had the ability to detect changes to dynamic environments and coordinate vehicle activities for goal achievement, in deploying a fleet of autonomous guided vehicles (AGVs) for material handling in an automated warehouse. Except [6] all mentioned work of multi-robot cooperation assumed the number of robots required for any particular task. In these approaches there was no coordination among the robots before the object transportation started. Consequently, these multi-robot systems were not fully autonomous.

Considerable work has been done to address the challenges of multi-robot coordination in the domain of robot soccer teams. Vail, et al. [8] proposed dynamic multi-robot coordination and presented a framework for task assignment and coordination for a group of robots in a soccer domain. They defined a bidding function to determine the suitability of a particular robot for a specific role. Iocchi, et al. [9] suggested fault tolerant distributed coordination for a multirobot system that is based on dynamic role assignment. Their method was based on broadcast communication of utility functions to define the role of a robot in a RoboCup team.

\section{OVERVIEW OF AN IMMUNE SYSTEM}

An immune system is a network of cells, tissues, and organs that act together to defend the body against foreign invaders called antigens. The secret of its success is an elaborate and dynamic communication network. Millions of these cells are organized into sets and subsets that pass information back and forth. Once the immune system is stimulated by a foreign substance, it undergoes tactical changes, produces powerful chemicals and rushes to troubled area to kill or neutralize the invader. The organs of the immune system, called lymphocytes, are positioned throughout the body. There are two major players in a human immune system: B-cells and Tcells. Only the B-cells are discussed here, as needed in the present work. B cells work by secreting substances called antibodies into the body fluid. As shown in Fig.1 (a), the structure of an antibody takes the form of letter "Y" which contains two chains termed light and heavy. Heavy chain represents the class of antibody whereas light chain is antigenically distinct $[10,11]$.

Antibodies contain a receptor called paratope, which can only bind to specific antigens. The binding region of the antigen, which is also known as antigenic determinant, is called

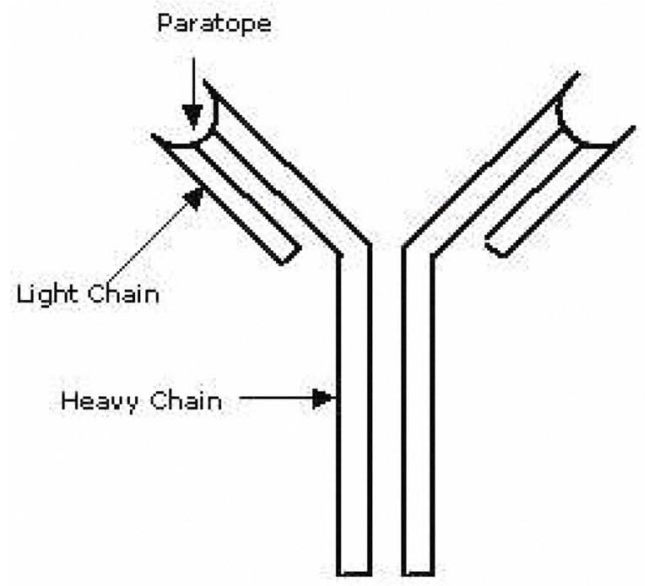

(a)

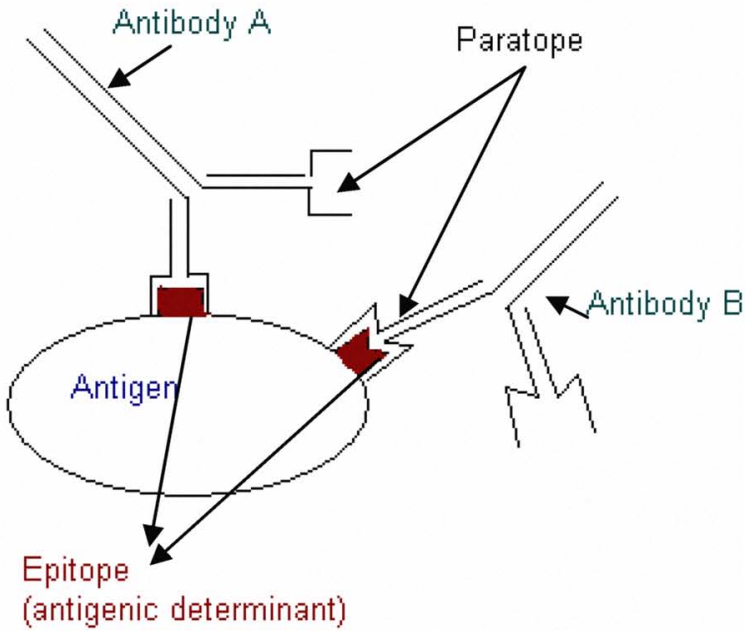

(b)

Fig.1. (a) Structure of an Antibody; (b) Cooperation of Antibodies to Kill or Neutralize an Antigen.

epitope as indicated in Fig.2 (b). Once antigens enter human body, B-cells are stimulated and the antibodies that have the capability of paratope complement epitope ambush antigens circulating in the blood stream [12]. The capability of an antibody to kill or neutralize the antigen depends upon the binding affinity, i.e., the degree of the binding of cell receptor paratope with the antigen's epitope. A paratope may not necessarily complement an epitope completely, resulting in weak binding affinity. Nevertheless it can help neutralize or kill an antigen with the help of another antibody. The greater the binding affinity, the higher are the chances to destroy the antigen. Furthermore, as shown in Fig.1 (b), it is also quite possible that one antibody may not destroy the antigen; in this case more antibodies can help and cooperate to kill the antigen. The recognition of an antigen by an antibody will stimulate proliferation and differentiation of the cells that produce matching clones or antibodies. These clones get priority when exposed to similar antigens, which leads to rapid immune response. It follows that the human immune 
system works cooperatively in a coordinating manner to achieve its task to eliminate an antigen.

\section{MULTI-ROBOT COORDINATION SYSTEM FOR OBJECT TRANSPORTATION}

\section{A. Problem Definition and Test Bed}

The system developed in the present work comprises three autonomous robots which are employed to transport an object to its goal location. Since the robots do not have knowledge about the physical parameters of the object, a robot at first will attempt to transport the abject by itself provided that it has the capabilities. If not, it will communicate and coordinate with other robots to get the assistance of the most suitable and capable robot. An experimental platform developed by us for this purpose is shown in Fig.2.

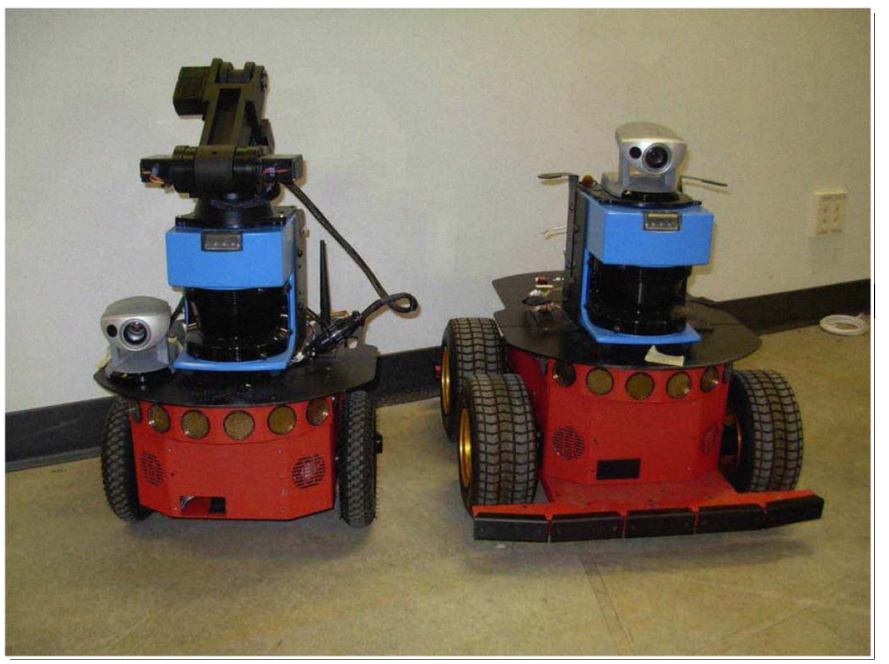

Fig.2. Test Bed for Multi-Robot Coordination.

Our test bed comprises three ActiveMedia Pioneer robots; two of which are Pioneer P3-DX models and the remaining one a Pioneer 3-AT model. The rugged P3-DX robot has a $44 \mathrm{~cm} \times 38 \mathrm{~cm} \times 22 \mathrm{~cm}$ aluminum body with two $16.5 \mathrm{~cm}$ drive wheels. It includes wireless Ethernet, eight sonar sensors, pantilt-zoom camera, gyro, and laser range finders. Each of these two robots houses a Pentium based computer running windows. P3-AT robot has a $50 \mathrm{~cm} \times 49 \mathrm{~cm} \times 26 \mathrm{~cm}$ fourwheeled out door base, also contains the same type of sensors as the other units but is more powerful, with the ability to carry a payload up to $30 \mathrm{~kg}$.

\section{B. Multi-Robot Coordination and Artificial Immune System (AIS)}

As mentioned earlier the structure of an antibody is similar to letter "Y", which comprises light and heavy chains that determine the capability and suitability of the particular antibody to tackle an antigen. In the present work, an approach inspired by the human immune system is applied to the multi-robot coordination system to determine if a particular robot is available and capable to handle the task at hand. As shown in Fig.3, the light chain represents the state of a robot. Some of the states are: "explore," "object recognition", and "busy." The heavy chain represents the capabilities of a robot to handle the particular object. The capabilities of a robot may include its sensors, payload capability, and mobility, as required to handle the object for transportation.
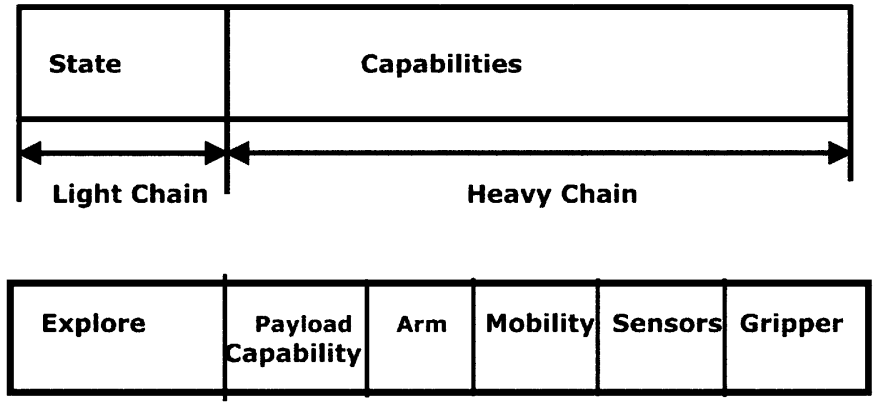

Fig.3. Availability and Capability Chain.

Once the availability and capability chains are satisfied then the binding affinity of different robots are calculated to select the most suitable robots among the fleet for assistance in the task. The binding affinity function is explained in Section D.

\section{Algorithm}

The mechanism for multi-robot coordination using AIS is as follows:

1. Each robot explores the surrounding environment within the sensory range. It is stimulated once it locates the object

2. The robot compares its own capabilities with the capabilities required to tackle the object. If it has the required capability it will try to transport the object alone. Else it will seek the help of the most capable robot.

3. If a robot is unable to transport the object alone it will broadcast a help signal to other robots. The signal includes the capabilities required to transport the object. The robot which seeks help is called the initiating robot.

4. Every robot who is in the explore state and within the communication range will calculate the binding affinity.

5. The robot with the highest binding affinity will come for help.

6. If a robot fails during assistance or coordination stages, another robot will be called for help.

\section{Binding Affinity Function}

The binding affinity determines the most suitable and capable robot that is available for help among the fleet of robots.

Binding affinity is a combination of distance between the 
robot and the help location $\left(d s_{m n}\right)$, success rate $\left(s r_{m n}\right)$, orientation of the robot $\left(o r_{m n}\right)$, and obstacles in the path between the robot and the help location if they are in the sensory range $\left(o b_{m n}\right)$.

Suppose that there are $m$ numbers of robots:

$R=\left\{r_{1}, r_{2}, r_{3}, \ldots . r_{m}\right\}, \quad$ where $\quad m=1,2, \ldots, y$

and there are $n$ number of tasks:

$T=\left\{t_{1}, t_{2}, t_{3}, \ldots . t_{n}\right\}$,

where $n=1,2, \ldots, z$

Binding Affinity is a function of:

$\beta A_{m n}=f\left(d s_{m n}, s r_{m n}, o r_{m n}, o b_{m n}\right)$

$\beta A_{m n}=w_{1}\left(d s_{m n}\right)^{-1}+w_{2}\left(s r_{m n}\right)+w_{3}\left(o r_{m n}\right)+w_{4}\left(o b_{m n}\right)$

where $d s_{m n}$ is the Euclidean distance between robot $m$ and task or help location $n$, as given by:

$d s_{m n}=\sqrt{\left(x_{m}-x_{n}\right)^{2}+\left(y_{m}-y_{n}\right)^{2}}$

Also, $s r_{m n}$ are the numbers of successful transportation of the particular object by any robot. If the robot transports the particular object, the success rate value is increased by one. Analogous to a human immune system, the robot will stand a better chance again to transport the object as it has already proved its capabilities with respect to the specific object. Furthermore, $o r_{m n}$ is the orientation of the robot, given by the value of angle $\theta$ between the robot heading and the task or help location; and $o b_{m n}$ are the obstacles in the path between the robot and the task or help location, provided they are in the detection range.

The concept can be explained through Fig 4. Since the robots have eight sonar sensors scanning $180^{\circ}$, it is divided into eight different segments each representing the scanning area of every sonar. If there is an obstacle within the sensory range corresponding segment is represented by binary 1 ; otherwise it is 0 . Note that $o b_{m n}$ is an eight-bit number. $w_{1}, w_{2}, w_{3}, w_{4}$ are the weights for the variables.

\section{E. Autonomous Fault Tolerant Multi-Robot Coordination}

Based on the function of a biological immune system, a new algorithm designed for multi-robot coordination, which is fault tolerant and completely autonomous. Suppose that there are " $m$ " robots and " $n$ " tasks. The robots are in the "explore" state in the beginning, while searching for a task.
Once it locates the task the robot compare its own capabilities

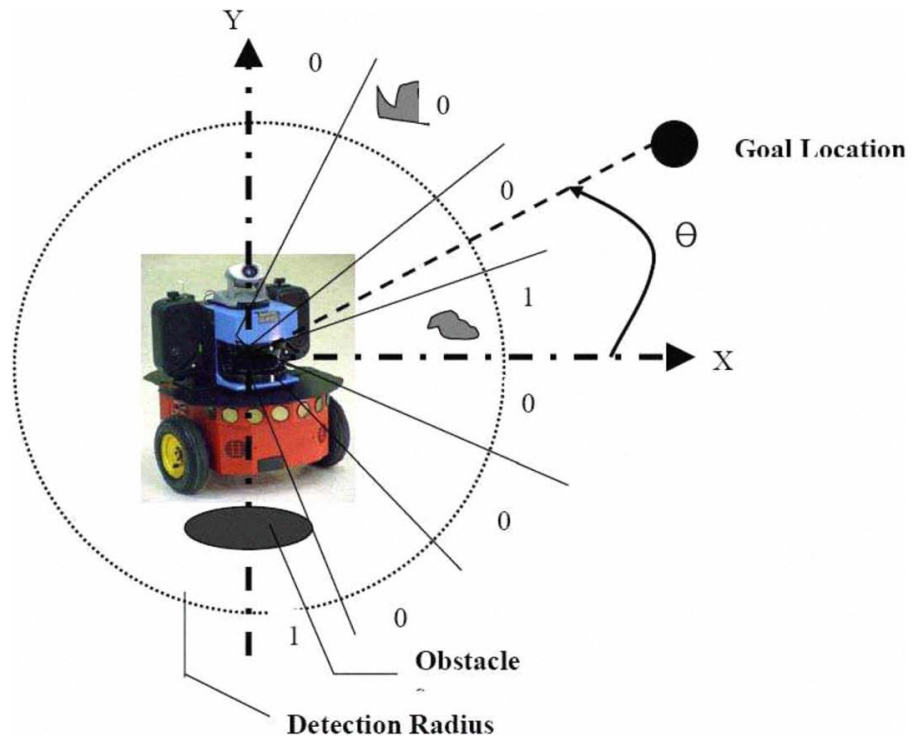

Fig.3 Orientation and Obstacle Detection within the Detection Radius.

with the capabilities that are required to transport the object, assuming that the task complexity is known. If the required capabilities for the task are not in the robot's capability list, which is the heavy chain, it will broadcast the task to other robots and will start searching for another task. Otherwise, if it has the capabilities, like a human it will try to transport the object by itself. If the initiating robot cannot transport the object alone, it will broadcast a help signal containing a heavy chain, indicating the capabilities required to transport the object. Only those robots in the communication range who are in the explore state will be stimulated with the signal and the rest will reject it. If more than one robot is available for help, every available robot will compare its heavy chain with the received heavy chain. If a robot has the necessary capabilities it will calculate its binding affinity using the binding affinity function and will broadcast it to the other robots. Every robot compares its own binding affinity with others, and the robot with the highest binding affinity will respond to help and the rest will go into the explore state again searching for another task.

During coordination, the helping robot calculates its binding affinity from time to time and sends it to the initiating robot unless it has reached the location where help is not required. The reason for calculating and sending the value of binding affinity after some specific time is to establish its own operational condition. If the helping robot fails partially; for example its motors fails to operate, the binding affinity will not increase. The helping robot will come to know about its failure and it will immediately stop communicating with the initiating robot. On the other hand if the initiating robot does not receive the value of the binding affinity from the helping robot or if the binding affinity is not in the increasing trend, it assumes that the helping robot has failed and will broadcast the help signal again. On the other hand if the initiating robot 
fails at any stage, it will not cripple the system as when the helping robot reaches the task or the help location. It will try to transport the object. Failing, the robot will broadcast the help signal and will become initiating robot.

\section{EXPERIMENTAL VALIDATION}

The approach presented in this paper has been validated with regard to fault tolerant coordination, task allocation, and system autonomy, through a series of experiments using the system of mobile robots in our laboratory. Numerous experiments were conducted for three different situations, details of which are given below.

In the first experiment, the robot which lacks capabilities to tackle a specific colored object successfully broadcasts the required capabilities to the other robots and goes into the explore state for other tasks.

In the second experiment, as indicated in Fig. 4, the robot DX1 reaches a specific colored object to be transported. After matching its capabilities with those required for the task, it tries to transport the object by itself. Failing this, it broadcasts a help signal to other available robots. Every robot calculates its binding affinity and compares with those of the others. The robot with the highest binding affinity is declared the winner, and it is called to help the initiating robot.

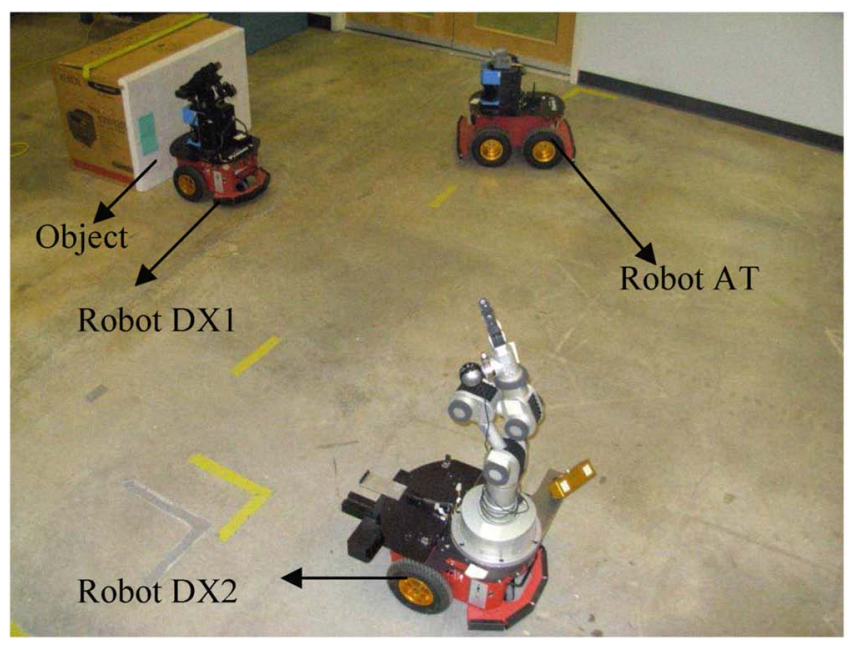

Fig.4. Robot AT Having the Highest Binding Affinity Moves Toward the Task to Help DX1.

The third experiment is shown in Fig. 5. Here, the robot DX1 reaches the colored object and fails to transport it alone even though the robot has the necessary capabilities. Then it broadcast the help signal. The winner robot based on highest binding affinity responds to help, but during its motion toward the help location, a software failure affects the helping robot AT. In the first set of experiments full failure was generated, and in the second set of experiments partial failures were generated. In both cases, the system recovers quickly from the failures. The initiating robot, in response to the failure in AT, broadcasts a help signal again. Robot DX2 proceeds to help DX1 as a result.

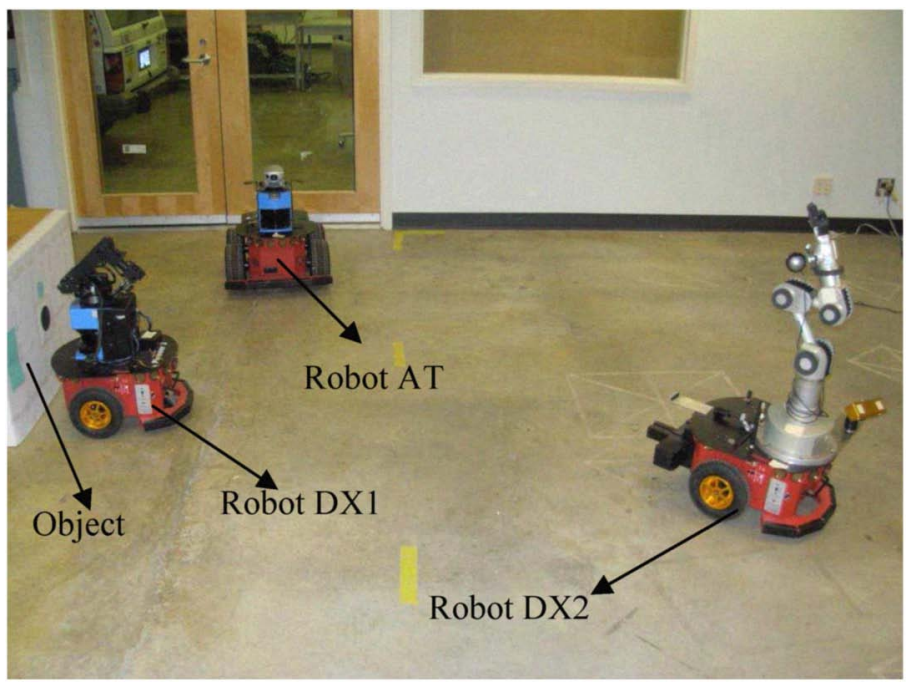

Fig.6. Failure Occurs in Robot AT During Its Motion to Help Robot DX1. Consequently Robot DX2 Moves to Help DX1.

As shown in Fig.6, ten trials each of multi-robot coordination were conducted without any failure in the helping robot, and with full failure in the helping robot. Three trials were conducted generating partial failure in the helping robot. It is clear from the graph that in the absence of failure, the system works well and accomplishes the task in a short span of time. It also completed its task when the helping robot failed completely, but took more time. The system took far more time to complete the task when partial failure is generated in the helping robot. The reason for this is that the initiating robot has to make sure that the helping robot is failed before sending a help signal again, though the communication link of the helping robot still functions during partial failure.

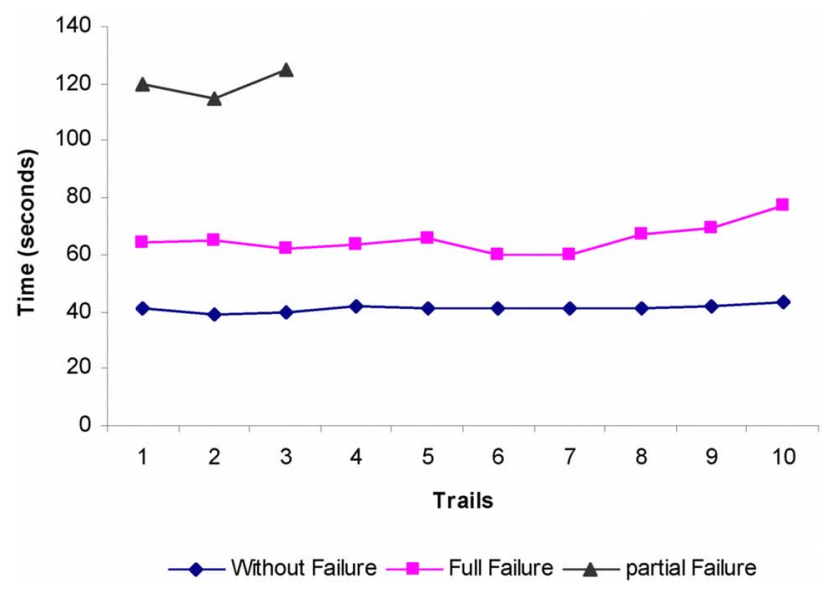

Fig.6 Trials of Multi-robot Coordination System: Without Failure; Full Failure; and Partial Failure.

\section{CONCLUSION}

A novel method of multi-robot coordination based on immune system was presented in this paper. The approach validated by implementing it on a team of physical robots in laboratory and 
carrying out extensive testing. It was demonstrated that the system was completely autonomous and could cope with all failures regardless of whether partial or full. The primary contribution of the paper pertains to distributed coordination.

As there is no leader in the robotic system, every robot would work independently and consequently, failures would not cripple the system. In this sense the developed system is autonomous and fault tolerant. Still there is a room for improvement in the system. The failure of a robot removes it from the team. However, it is quite possible that the failed robot may recover and become a member of the team once again. Also, if the velocity of the robot is included as well in the binding affinity, the system will become more efficient as. Specifically, a robot that satisfies all the variables of binding affinity but is very slow to move can be overtaken by a robot that is farther but able to reach the help location faster.

\section{REFERENCES}

[1] Maja J Mataric, Martin Nilsson, and Kristian T. Simsarian, "Cooperative multi-robot box-pushing," Proc. IEEE/RSJ Int.Conf on Human Robot Interaction and cooperative robots, Pittsburgh, PA, pp 556-561, 1995.

[2] Lynne E. Parker, "ALLIANCE: an architecture for fault tolerant, cooperative control of heterogeneous mobile robots," Proc. IEEE/RSJ/GI International Conference on Advanced Robotic Systems and the Real World, IROS, Munich, Germany, Vol. 2, pp 776-783, 1994.

[3] Lynne E.Parker, "ALLIANCE: An architecture for Fault Tolerant Multirobot Cooperation," IEEE Trans. Robotics and Automation, Vol. 14, No 2, pp. 220-240, April 1998.

[4] Ying Wang, and Clarence W. de Silva, "An object transportation system with multiple robots and machine learning," Proc. IEEE/ACC, Int Conf, Portland, OR, Vol. 2, pp 1371- 1376, June 2005.

[5] Jiming Liu, Jianbing Wu, and Xun Lai, "Analytical and experimental results on multiagent cooperative behavior evolution," Proc of IEEE/IEE congress on Evolutionary Computation," Washington, DC, Vol3, pp. 1732-1739, July 1999.

[6] Yunyuan Gao, and Wei Wei, "A new multi-robot self-determination cooperation method based on immune agent network," Proc of IEEE conf on Robotics and Automation, Barcelona, Spain, pp. 390 - 395, April 2005.

[7] Henry Y.K. Lau, Vicky W.K. Wong, and Ivan S.K. Lee "Immunitybased autonomous guided vehicles control," Applied Soft Computing, vol 7, Issue 1, pp $41-57$, January 2007.

[8] Douglas Vail and Manuela Veloso, "Dynamic Multi-robot coordination," Multi-Robot Systems: From Swarm to Intelligent Automata, vol II, pp.87-98, 2003

[9] Luca Iocche and Daniele Nardi, "Distributed coordination in heterogeneous multi-robot systems," J. Autonomous Robots, Vol 15, No 2, pp 155 - 168, September, 2003.

[10] Henry Y. K. Lau, and Vicky W.K. Wong, "An immunity-based distributed multiagent-control framework," IEEE Trans. System, Man, and Cybernatics- Part A: System and Humans, Vol. 36, No 1, pp 91 108, Jnuary 2006.

[11] L.J. Eales, Immunology for Life Scientists: A Basic Introduction, Wiley,, Chichester, U.K., 1997.

[12] http://www.niaid.nih.gov/Publications/immune/the immu ne system.pdf 